\title{
Kinder-Mittelalterbilder im Unterricht?
}

\section{Ein Kommentar zur didaktischen Relevanz und praktischen Umsetzbarkeit ${ }^{1}$}

Janina Lillge hat sich in ihrem Vortrag der Frage gewidmet, inwieweit Kinder-Mittelalterbilder als Möglichkeit und Herausforderung für Lehrende an Schulen gelten können.

Bereits zu Beginn wird konstatiert, dass trotz der vermeintlichen „Dauerkrise“ des Mittelalters und der sinkenden Bedeutung sowohl in der universitären als auch in der schulischen Lehre ein regelrechter Mittelalter-Boom im Bereich der Geschichtskultur festzustellen ist: in Filmen, Serien, Spielen, Werbung oder auch in Sachbücher für Kinder - das Mittelalter scheint omnipräsent. ${ }^{2}$ Doch wie kommt es zu dieser Divergenz, welche Folgen hat dies speziell für die präkonzeptionellen Mittelalterbilder von Kindern und wie kann hiermit in der universitären und schulischen Lehre umgegangen werden? Diese Fragen sollen folgend aufgegriffen und aus didaktischer Sicht diskutiert werden.

\section{Präkonzeptionelle Mittelalterbilder}

Wie Lillge darlegt, bilden sich bereits in früher Kindheit Präkonzepte in Bezug auf das Mittelalterbild aus. Diese basieren nach Lillge vor allem auf geschichtskulturellen Produkten wie Sachbüchern über Burgen und Ritter, Spiele, Spielzeuge sowie Filmen und Serien. Gleiches belegt auch eine Studie von Gerhild Löffler aus dem Jahr 2009: Löffler befragte Schüler*innen der 5. und 6. Klasse nach Interessen und Vorwissen in Bezug auf das Mittelalter, wobei viele Schüler*innen bereits vor der unterrichtlichen Behandlung des Mittelalters über ein breites Vorwissen sowie ein hohes Interesse am Mittelalter verfügten. Das Vorwissen rührte vor allem von populärhistorischen Erzählungen, beispielsweise aus Filmen, Märchen und Spielen, her. ${ }^{3}$

\footnotetext{
1 Kommentierter Beitrag: https://dx.doi.org/10.38072/2703-0784/p10.

2 Vgl. Hans-Werner Goetz, Moderne Mediävistik. Stand und Perspektiven der Mittelalterforschung. Darmstadt 1999, 7, 142145; Hiram Kümper, Materialwissenschaft Mediävistik. Einführung in die Historischen Hilfswissenschaften. Paderborn $2014,11$.

3 Vgl. Nicola Brauch/Gerhild Löffler, Die Wirklichkeit des Mittelalters in der Schulpraxis. Erfahrungsbericht und kompetenzdidaktische Überlegungen, in: Thomas Martin Bruck/Gerhild Löffler (Hrsg.), Das Mittelalter zwischen Vorstellung und Wirklichkeit. Probleme, Perspektiven und Anstöße für die Unterrichtspraxis, Münster 2011, 255-268, hier 257; ähnliche Ergebnisse finden sich bei Michael Sauer, Geschichte und Geschichtsunterricht - Erfahrungen und Interessen, in: Geschichte in Wissenschaft und Unterricht 4, 2006, 266-275.
}

\footnotetext{
>> Das Werk ist unter der Creative-Commons-Lizenz Namensnennung - Weitergabe unter gleichen Bedingungen 4.0 International veröffentlicht. Den Vertragstext finden Sie unter: https://creativecommons.org/licenses/by-sa/4.0/deed.de. Bitte beachten Sie, dass einzelne, entsprechend gekennzeichnete Teile des Werks von der genannten Lizenz ausgenommen sein bzw. anderen urheberrechtlichen Bedingungen unterliegen können.
} 
Diese in der Kindheit ausgebildeten Mittelalterbilder erweisen sich nach Lillge als stabile und äußerst resistente Konzepte, welche sich gleichwohl bei Studierenden als auch bei Erwachsenen wiederfinden lassen und häufig weder differenziert noch wissenschaftlich korrekt sind. Aus diesem Befund folgert Lillge, dass die kindlichen Präkonzepte im Geschichtsunterricht keine nachhaltige Veränderung erfahren und benennt die Gefahr, dass unreflektierte und inkorrekte Mittelalterbilder auch für künftige Generationen weitertradiert werden. Diese Gefahr findet sich ähnlich in der aktuellen Forschung zum Sachunterricht bestätigt: Auch wenn noch nicht endgültig geklärt ist, ob Präkonzepte aus kohärenten und theorieähnlichen Strukturen bestehen oder ob es sich um unstrukturierte und nicht verknüpfte Wissensbestandteile handelt, so ist nach Marco Adamina u. a. „[u]nstrittig und empirisch belegt [...], dass Präkonzepte eine hohe Resistenz gegen Veränderungen aufweisen und dass durch Unterricht nur selten ein [...] Konzeptwechsel provoziert werden kann“". Kornelia Möller betont den konstruktivistischen Ansatz des Lernens und führt hierzu aus, dass Lernende ihr Wissen und ihre Vorstellungen selbst aufbauen, diese folglich vom lernenden Subjekt aktiv konstruiert bzw. rekonstruiert werden müssen und „es sich [hierbei] um eine Differenzierung, Erweiterung oder um einen Neuaufbau bzw. eine Umstrukturierung von Vorstellungen handeln kann“. ${ }^{5}$ Umso wichtiger ist es, dass Unterricht auf das Vorwissen und die Präkonzepte aktiv eingeht, um diese zu reflektieren und einen Konzeptwechsel zu ermöglichen.

Auch Lillge erkennt hier eine Chance, eben diese Präkonzepte als Ausgangpunkt für einen reflektierten Umgang mit Mittelalterbildern im Unterricht nutzbar zu machen. Dieser Ansatz soll im Folgendem in seiner Realisierbarkeit und in seinem didaktischen Mehrwert diskutiert werden.

\section{Curriculare Voraussetzungen}

Als erstes ist hierfür ein Blick auf die curricularen Voraussetzungen zu werfen. Beginnt man mit dem Sachunterricht in der Grundschule, so ist festzustellen, dass die aktuellen Fachanforderungen des Landes Schleswig-Holstein eine Behandlung des Mittelalters nicht vorschreiben, der Begriff des Mittelalters überdies gar keine Nennung erfährt. ${ }^{6}$ Dieser Umstand könnte bei manchem Rezipienten Empörung hervorrufen. Immerhin

4 Marco Adamina/Markus Kübler/Katharina Kalcsics/Sophia Bietenhard/Eva Engeli, Vorstellungen von Schülerinnen und Schülern zu Themen des Sachunterrichts und des Fachbereiches Natur, Mensch, Gesellschaft - Einführung, in: Dies. (Hrsg.), „Wie ich mir das denke und vorstelle...". Vorstellungen von Schülerinnen und Schülern zu Themen des Sachunterrichts und des Fachbereiches Natur, Mensch, Gesellschaft, Bad Heilbrunn 2018, 7-20, hier $7 \mathrm{f}$.

5 Kornelia Möller, Die Bedeutung von Schülervorstellungen für das Lernen im Sachunterricht, in: Marco Adamina/Markus Kübler/ Katharina Kalcsics/Sophia Bietenhard/Eva Engeli (Hrsg.) , "Wie ich mir das denke und vorstelle..." (wie Anm. 4), 35-50, hier 38.

6 Vgl. Ministerium für Bildung, Wissenschaft und Kultur des Landes Schleswig-Holstein (Hrsg.), Fachanforderungen Sachunterricht Primarstufe/Grundschule, Kiel 2019. 
bilden Fachanforderungen und Lehrpläne, wie Arnold Bühler ausführt, „,bekanntlich die conditio sine qua non".7 Bedeutet: Themen, welche nicht in den Fachanforderungen verankert sind, finden ihren Weg nur schwerlich in die gängigen Unterrichtswerke und somit auch nur schwerlich in die Klassenzimmer zu den Lernenden. Doch von einem blinden Aktionismus, die Fachanforderungen erneut durch zahlreiche, verpflichtende Inhalte zu überfrachten, sei an dieser Stelle gewarnt. ${ }^{8}$ Nicht nur würde eine solche Änderung bei vielen Praktiker*innen Herzrasen auslösen, da noch mehr Inhalte in kurzer Zeit zu vermitteln wären. Auch würde es dazu führen, dass die Unterrichtsgestaltung weniger flexibel wäre und den Lehrenden auch in gewissem Maß die Freiheit genommen wäre, auf individuelle Interessen sowie flexibel auf die Lernenden und ihren Denk- und Lernprozess eingehen zu können. Dies wäre vor dem Hintergrund der Subjektorientierung ein fatales Signal und als enormer Rückschritt zu bewerten: Nach der subjektorientierten Geschichtsdidaktik steht nicht primär die Vermittlung von Inhalten oder gar eine Diskussion über den Kanon im Vordergrund, sondern die „individuellen mentalen Strukturen, also die Vorstellungen über Vergangenheit und Geschichte sowie die sie strukturierenden Konzepte“ ${ }^{\text {“9 }}$ der lernenden Subjekte. Subjekte sind hierbei als lernende Individuen zu verstehen, ihr historisches Denken ist unter anderem durch ihre Interessen, Vorerfahrungen, aber auch Gefühle geprägt. Aus diesem Grund sind der Individualisierung und der Differenzierung eine hohe Bedeutung einzuräumen. ${ }^{10}$ Folgend sollten sich Fachanforderungen zeitgemäß zunehmend von Inhalten lösen, Lernende als erkennende Subjekte anerkennen und dadurch historisches Lernen und Denken fördern. Dies bedeutet gleichwohl nicht, dass das Mittelalter in den Fachanforderungen des Sachunterrichts in Schleswig-Holstein gar keine Möglichkeit der Thematisierung findet: das Themenfeld "Zeit und Entwicklung“, welches die Behandlung der Geschichte des eigenen Wohnortes oder des Alltags in einem Vergleich zwischen „früher“ und "heute" vorsieht, bietet hier eine Chance. ${ }^{11}$

Die Fachanforderungen des Faches Geschichte schreiben hingegen eine verbindliche Behandlung des Mittelalters vor. So ist für die Sekundarstufe I „Das Mittelalter - eine finstere Zeit?“ als ein einzelner Themenbereich aufgeführt. Inhaltlich sollen hier insbesondere die „Lebens- und Herrschaftsformen im Mittelalter“ sowie „Juden, Christen und Muslime“ Beachtung finden. Überdies wird als fachmethodischer Schwerpunkt „Lernen am anderen Ort“ aufgeführt, als Beispiel sogar der Besuch eines Mittelaltermarktes genannt, anhand dessen sich die Mittelalterbilder der

7 Arnold Bühler, „Verordnete Finsternis“. Mittelalter nach Lehrplan, in: Thomas Martin Buck/Gerhild Löffler (Hrsg.), Das Mittelalter zwischen Vorstellung und Wirklichkeit (wie Anm. 3), 245-254, hier 246.

8 Vgl. ebd., 246.

9 Christoph Kühberger, Subjektorientierte Geschichtsdidaktik. Eine Annäherung zwischen Theorie, Empirie und Pragmatik. In: Heinrich Ammerer/Thomas Hellmuth/Christoph Kühberger (Hrsg.): Subjektorientierte Geschichtsdidaktik, Schwalbach/Ts. 2015, 13-47, hier 41.

10 Vgl. ebd., 41.

11 Vgl. Fachanforderungen Sachunterricht (wie Anm. 6), 28. 
Lernenden thematisieren ließen. Auch für die Sekundarstufe II lassen sich zum einen die „Christliche und islamische Welt“ als fakultativer Inhalt sowie der „Feudalismus“ als verbindlicher Inhalt festhalten, ${ }^{12}$ welche beide die Chance bieten, gängige und populäre Mittelalterbilder zu thematisieren, beispielsweise durch die Analyse einzelner Sequenzen des Spielfilms „Königreich der Himmel“. Spielfilme als Gegenstand der Geschichtskultur können hier im Besonderen Maß dazu beitragen, die narrative Kompetenz zu schulen und vorhandene Narrationen zu dekonstruieren sowie auf die jeweilige Intention hinter Spielfilmen hinzuweisen. ${ }^{13}$

\section{Kompetenzorientierung}

In Bezug auf die curricularen Voraussetzungen und der inhaltlichen Verortung steht es folglich nicht allzu schlecht um das Mittelalter. Doch geht es im Geschichtsunterricht nicht vornehmlich um die Vermittlung von Inhalten. Seit den 1960ern wurde zunehmend der Fokus auf fachliche Fähigkeiten und Fertigkeiten gesetzt, welche nunmehr mit der Kompetenzorientierung auch als Prinzip verankert sind. ${ }^{14}$ In den Fachanforderungen des Faches Geschichte lautet dies wie folgt: „Geschichtsunterricht zielt auf den Erwerb fachlicher Kompetenzen in Auseinandersetzung mit historischen Inhalten. Kenntnisse von geschichtlichen Ereignissen und Entwicklungen sind eine notwendige, nicht jedoch eine hinreichende Voraussetzung historischen Lernens. Übergeordnetes Ziel des Geschichtsunterrichts muss daher die Entwicklung einer narrativen Kompetenz sein, die es den Schüler*innen ermöglicht, durch historisches Erzählen Sinn über Zeiterfahrung zu bilden. " ${ }^{15}$ Eben in dieser Zielsetzung bietet sich eine Möglichkeit, das Mittelalter sowie seine mehr oder weniger bunten, differenzierten und komplexen Repräsentationen im Kopf der Lernenden aufzugreifen.

Im Folgenden soll dies anhand eines kurzen Beispiels, in dem wir erneut auf den bereits genannten außerschulischen Lernort ,Mittelaltermarkt' der Sekundarstufe I eingehen, dargestellt werden. Die narrative Kompetenz als Ausdruck eines reflektierten Geschichtsbewusstseins sowie der Fähigkeit, historisch zu denken, setzt sich aus vier Teilbereichen zusammen: Der Wahrnehmungs-, Erschließungs-, Sachurteils- und Ori-

\footnotetext{
12 Ministerium für Bildung, Wissenschaft und Kultur des Landes Schleswig-Holstein (Hrsg.), Fachanforderungen Geschichte. Allgemein bildende Schulen, Sekundarstufe I, Sekundarstufe II. Kiel 2016, 20, 28.

13 Zu dem genannten Format lassen sich sowohl wissenschaftliche Literatur als auch Unterrichtsideen finden, hier beispielhaft zu nennen: Sandra Pfistermüller, Deus lo vult. Die Kreuzzugsthematik in „Königreich der Himmel“, in: Christian Rohr (Hrsg.), Alles heldenhaft, grausam und schmutzig? Mittelalterrezeption in der Populärkultur. Wien 2011 (Austria. Forschung und Wissenschaft. Geschichte, Bd. 7), 159-170; sowie Unterrichtsmaterial https://www.uni-giessen.de/fbz/fb04/institute/ geschichte/didaktik/Alte\%20Seiten/uebertrag-didaktik/gott-will-es (12.10.2020).

14 Vgl. Michele Barricelli/Peter Gautschi/Andreas Körber, Kompetenzen und Kompetenzmodelle im Geschichtsunterricht, in: Michele Barricelli/Martin Lücke (Hrsg.) Handbuch Praxis des Geschichtsunterrichts (2. Aufl.). Schwalbach/Ts. 2017, 207-235, hier 207f.

15 Fachanforderungen Geschichte (wie Anm. 12), 15.
} 
entierungskompetenz. ${ }^{16}$ Der Besuch eines Mittelaltermarktes lädt die Lernenden dazu ein, einen Aspekt der Geschichtskultur aktiv wahrzunehmen sowie eine kognitive Dissonanz zu erzeugen, welche in einer Frage oder Hypothese münden kann (Wahrnehmungskompetenz). Diese Eindrücke und Fragen, welche sich aus einem Besuch eines Mittelaltermarktes ergeben, können im Unterrichtsgeschehen aufgegriffen werden, indem man diese gemeinsam verbalisiert und Lösungswege zur Beantwortung der Frage sucht. Zur Überprüfung der Frage oder Hypothese gilt es Quellen oder Darstellungstexte zu erarbeiten, auszuwerten (Erschließungskompetenz) sowie die vorliegende Sachanalyse zu einem Sachurteil zu bündeln (Sachurteilskompetenz). Anschließend sind die Lernenden auf einer metakognitiven Ebene gefordert, indem sie auf Basis der Erkenntnisse ihr Mittelalterbild als individuelle Repräsentation von Geschichte erkennen, hinterfragen, bewerten und ggf. anpassen, um schlussendlich ihr Geschichtsbewusstsein zu erweitern (Orientierungskompetenz).

Das Kompetenzmodell nach Gautschi erfüllt somit die allgemeine Zielsetzung, Lernende zur Urteils- und Diskursfähigkeit zu befähigen und sie zur Partizipation an unserer demokratischen Gesellschaft zu befähigen. ${ }^{17}$ Eben diese Fähigkeit zur Bewertung von Diskursen, die Einordnung dieser in ihren historischen Kontext und die Erkenntnis über die jeweiligen Intentionen, fordert auch Lillge und weist hier mit Recht auf das Potenzial von Geschichtskultur und im Besonderen von Mittelalterbildern hin. Diese können exemplarisch genutzt werden, um die Diskursfähigkeit der Lernenden in einem geschützten Raum wie der Schule zu üben, eine Fähigkeit, welche in Zeiten von Fake-News und Verschwörungstheorien auch über den Schulunterricht hinaus ungemein wichtig ist. ${ }^{18}$

Ein solcher Geschichtsunterricht, der aktuelle Diskurse und geschichtskulturelle Aspekte aus der Lebens- und Erfahrungswelt von Schüler*innen aktiv und kontinuierlich einbindet, würde zum einen nach dem Erziehungswissenschaftler Wolfgang Klafki nicht nur die Zugänglichkeit zu einem Thema fördern, sondern auch die Gegenwarts- und Zukunftsbedeutung für die Lernenden verdeutlichen. ${ }^{19}$ Ebenfalls dienen aktuelle Diskurse zur Erweiterung des Geschichtsbewusstseins, welches Jeismann als eine dynamische Größe beschreibt, in der die Zeithorizonte der Vergangenheitsdeutung, Gegenwartserfahrung und Zukunftserwartung miteinander verschmelzen. ${ }^{20}$ Ob man durch einen solchen Ansatz dem häufig beklagten fehlenden deklarativen

16 Vgl. Fachanforderungen Geschichte (wie Anm. 12), 15.

17 Vgl. ebd., 26.

18 An dieser Stelle sei lediglich das Beispiel der sogenannten „QAnon-Bewegung" genannt, welche vor allem in den sozialen Netzwerken ihre Verschwörungstheorien verbreitet und so unreflektiert von Lernenden konsumiert werden können, hierzu Jan Vollmer, QAnon ist die perfekte Verschwörungstheorie für das Internet, in Welt (9.9.2020), https://www.welt.de/wirtschaft/webwelt/article215329520/QAnon-ist-die-perfekte-Verschwoerungstheorie-fuer-das-Internet.html (letzter Zugriff: 26.5.2021).

19 Vgl. https://www.uni-due.de/Ev-Theologie/courses/course-stuff/didaktik-fragen.htm (13.10.2020).

20 Bernd Schönemann, Geschichtsdidaktik, Geschichtskultur, Geschichtswissenschaft, in: Hilke Günther-Arndt (Hrsg.): Geschichtsdidaktik. Praxishandbuch für die Sekundarstufe I und II. (2. Aufl.). Berlin 2005, 11-22, hier $12 f$. 
Wissen von Schüler*innen, folgend auch von Student*innen, entgegenwirken könnte, bleibt an dieser Stelle jedoch offen. ${ }^{21}$

\section{Fazit}

Zusammenfassend ist die Forderung von Janina Lillge, (Kinder-)Mittelalterbilder sowie in diesem Zusammenhang geschichtskulturelle Aspekte verstärkt im Schulunterricht sowie auch in der universitären Lehre einzubinden und das Potenzial dieser zu nutzen, voll zu unterstützen. Der von Lillge skizzierten Gefahr, dass sich unreflektierte, undifferenzierte und unwissenschaftliche Geschichtsbilder in der Lehre und unserer Gesellschaft verfestigen, wäre anderenfalls zuzustimmen. Von einer Reform der Fachanforderungen und deren verbindlichen Inhalte ist aus genannten Gründen jedoch abzusehen, aber dennoch würde es sich anbieten, verstärkt exemplarisch zu arbeiten und vor allem die Lebens- und Erfahrungswelt der Schüler*innen in Form der Geschichtskultur noch mehr mit einzubeziehen. Auch bedarf es weiterer Forschung, vor allem im Bereich der kindlichen Mittelalterbilder, um umfassendere Erkenntnisse über die Ausbildung von Präkonzepten sowie deren Veränderbarkeit zu gewinnen. Für den Geschichtsunterricht ist hier weiterhin wichtig, dass die diagnostischen Fähigkeiten von Lehrenden geschult werden, denn erst wenn sie die individuellen Präkonzepte ihrer Schüler*innen erfassen können, können Konzepte gefördert werden. Für die Hochschuldidaktik bedeutet dies, dass Lernende sich ihrer zukünftigen Rolle als Lehrpersonen bewusst werden und ihre eigenen Stereotype und Vorurteile infrage stellen müssen. ${ }^{22}$ Universitäre Lehrveranstaltungen, wie die von Janina Lillge skizzierte Übung „Ritter und Prinzessin? Mittelalterbilder von und für Kinder“, tragen einen wichtigen Teil hierzu bei.

\section{Autorin}

\section{Svantje Kühl}

Studienrätin und Lehrbeauftragte im Bereich Didaktik der Geschichte an der Christian-Albrechts-Universität zu Kiel. Ihre Forschungsschwerpunkte sind Emotionen im Geschichtsunterricht sowie Geschichtskultur.

skuehl@histosem.uni-kiel.de

21 Nicola Brauch, Die vergessenen Fragen an die mittelalterliche Geschichte, in: Johannes Grabmayer (Hrsg.), Das Bild vom Mittelalter. Klagenfurt 2013 (Schriftenreihe der Akademie Friesach. Neue Folge, Bd. 3), 169; Nicola Brauch/Gerhild Löffler, Die Wirklichkeit des Mittelalters (wie Anm. 3), 255.

22 An dieser Stelle gibt es in der Wirksamkeitsforschung zur Professionalisierung viele Hinweise darauf, dass dieser reflektierende Teil bezüglich der eigenen Ausbildung hochwirksam ist. Er wurde auch schon vorher theoretisch gefordert, siehe Donald Schön: The Reflective Practitioner: How Professionals Think in Action. New York 1983. 